fuel, cut out certain waste at the collieries and generally improve their efficiency, but the merits of such a scheme must rest on economic facts.

Prof. Baily bases his main comparison on stations working on a load factor of 40 per cent, but in future there will be two types of station in operation, the base load station working on a load factor of something of the order of 80 per cent and peak load stations operating on a poor load factor. Into which category are the pit-head stations to be put?

We think there may be individual cases where a pit-head station will compare favourably with an existing selected station, but we can see no substantial evidence to justify any general scheme which would reduce the number or render redundant the existing base load stations. As soon as it can be proved that the pit-head generating station is capable of supplying energy to the Grid at a price below that of the most modern stations now connected to it we believe that: (1) capital will be found for such a station; (2) the Central Electricity Board will be prepared to enter into a contract to purchase the whole of the station output; and (3) the Electricity Commissioners will give their consent.

\title{
International Conference on Physics
}

COME time ago, the Physical Society became $D$ convinced that results of value could be anticipated from an international conference on atomic (particularly nuclear) physics, and it was the intention of the Society to call such a conference this year. At the same time, the British National Committee for Physics-one of the constituent bodies which together form the International Union of Pure and Applied Physicsproposed to invite the Union to hold its next meeting in Great Britain.

It was a natural step to amalgamate these two functions, and the six-day meeting on October 1-6 has amply demonstrated the wisdom of that step. It was, we believe, the first occasion on which a meeting of the International Union had taken the form of a colloquium, and it brought together a most impressive array of physicists of note from many countries. The actual membership was nearly 600 , of whom some 150 came from abroad. The international nature of the conference was perhaps best illustrated when an Italian, speaking in French, gave to the mainly English audience an account of recent work by a German who was unable to attend in person. The meeting was held in London in the rooms of the Royal Society and at the Royal Institution, and in addition, at the invitation of Lord Rutherford, one session was held at the Cavendish Laboratory, Cambridge.

The more formal business of the International Union included the ratification of a report on symbols and definitions, with which we hope to deal later, and the election of Prof. Niels Bohr to succeed Prof. R. A. Millikan in the presidential chair. In addition to this, the Union was responsible for the organisation of a discussion on certain problems of the solid state. In crystals, there is much evidence tending to show that, over and above the lattice regularity revealed by $\mathrm{X}$-rays, there is a further definite structure, on a larger scale. The existence of such a block or mosaic structure was much debated, but assuming it to exist, it becomes a question of great interest to decide whether it is present inevitably, as a consequence of the need for the potential energy to become a minimum, or whether it is fortuitous, and due to something in the nature of flaws distributed statistically. The theoretical question involved here was discussed in one form or another at several of the meetings, and it seems likely that a solution will at least be expedited by the interplay of ideas and by consideration of the numerous suggestions made.

The most immediately obvious point about crystals is their lack of tensile strength, as compared with the value to be expected on theoretical grounds. The difference for rock-salt, for example, is about a thousand-fold, and two rival theories are in existence to account for this. One theory is that outlined above; the other locates the weaknesses at cracks on the surface of the specimen. This crack theory receives strong support from many experiments where surface treatment alone has been found to alter the tensile strength considerably ; perhaps the most striking illustration is the discovery by Joffé and others, that the strength of rock-salt is increased twentyfive fold by merely carrying out the experiments in hot water. Of course, it must be borne in mind that a block structure might still exist, even if not needed to explain this particular fact. Much of the evidence for a block structure rests on data obtained by studies of plastic yielding, as well as on chemical facts and microscopic examination.

That part of the conference for which the Physical Society was directly responsible concerned itself with certain aspects, mainly experimental, of the recent advances in nuclear physics. It is true that fifteen years have elapsed since Lord Rutherford first succeeded in demonstrating that certain nuclei could be disintegrated by 
bombardment with sufficiently swift $\alpha$-particles, but at that time the products observed were always protons. We thought that nuclei were made up of protons and (negative) electrons. A few years ago, nuclear reactions were studied, using as projectiles not $\alpha$-particles, but protons accelerated by means of intense electric fields produced for the purpose. This development was rapidly followed by the use of the newly discovered neutrons as bombarding particles, leading again to further nuclear reactions. Meanwhile, spectroscopy had given clear evidence of the actual existence of the hydrogen isotope of mass 2 , and it was not long before this was separated, and its ions used as bombarding particles. At the same time, it was realised that the cosmic radiation with which space appears to be permeated, whether it is corpuscular or of wave-character, forms a powerful source with which Nature is continually carrying out disintegration experiments. Thus we may bombard any element with protons, deutons, neutrons, photons (including $\gamma$-rays) or $x$-particles, and may as a result obtain any one or more of these, either with or without ordinary negative electrons.

Even this, however, does not exhaust the list of particles. In certain reactions, the positive electron appears as a product. This is most clearly demonstrated in Wilson cloud-chamber photographs taken in magnetic fields, where the curvature of the track gives immediate evidence of the sign of the charge, whilst the nature of the track makes its electronic character evident. This particle is emitted as a product by the new radioactive elements discovered recently by $\mathbf{M}$. and Mme. Joliot. These elements, which have lives varying from less than a minute to a few hours, are produced from ordinary elements by bombardment with neutrons, and there is no reason to suppose that the list of them is by any means complete. Indeed, Fermi has given reasons for supposing that, by bombarding uranium with neutrons, he has succeeded in producing a new element which is neither uranium itself, nor any of the five or six which immediately precede it in the periodic table. Consequently he suggests that it may be the element of atomic number 93 or 94 , until now unknown.

As to the reactions which may occur in these various bombardments, it seems, as pointed out by Lord Rutherford, that practically any transmutation occurs (though the probabilities of the different reactions naturally differ) provided only that it is consistent with the energy laws. In this connexion, of course, a knowledge of the so-called 'mass-defects' is of primary importance, since they are so intimately related to the energy changes, in consequence of the Einstein law $E=m c^{2}$.
Among these mass-defects, many are known with great accuracy from measurements with the massspectrograph or otherwise, but there is one particle, the neutron, for which the value of the mass. defect is uncertain.

This uncertainty is particularly regrettable since the values suggested lie on opposite sides of the mass of the proton, and consequently the relative stability of the two particles cannot properly be assessed. The higher of the two values put forward at the Conference depends on the assumption that, if one and the same element can be obtained as a product in two different reactions with the same initial reactants, then the energy content of that resultant is the same in both cases. Whilst the assumption is a reasonable one, yet the existence of certain isotopic isobars (isotopes with the same mass number) with different energy contents seems almost proved, when these are obtained in different reactions. If this can occur when the end-product arises from two different reactions, it may possibly turn out to be true that the isotopic isobars obtained by different mechanisms in a single reaction will also differ in energy, in which case the lower value for the mass of the neutron would hold the field.

It was suggested by Gamow at the Cambridge meeting of the Conference that the two forms of a single isotope referred to above might be explained if we could introduce another (not yet observed) particle, the negative proton, since in that case one form could contain a positive and a negative proton where the other contained two neutrons.

Even when we have added the negative proton to our list of particles, it is not complete. There is the 'neutrino', introduced by Fermi to maintain the conservation of energy, momentum and spin in $\beta$-ray emission. This particle, which is uncharged, has been discussed by several authors, all of whom conclude that its mass is much less than that of the electron.

Other papers, with which space does not permit us to deal, were concerned with the nature of the cosmic rays, which, besides being powerful agents in causing nuclear reactions, must of course be the products of such reactions, and are therefore to be studied also from that point of view.

In addition to the formal meetings, the sccial side was well cared for, and, further, many an informal discussion took place, which must certainly not be overlooked when we try to assess the scientific value of the Conference. All who attended seem agreed in hoping that another such meeting may be held in due course, and they will be well satisfied if it is equally successful.

J. H. A. 